\section{Correlação da Medida de Espessura Intra- Abdominal Medida pela Ultra-Sonografia com os Fatores de Risco Cardiovascular}

\begin{abstract}
RESUMO
O objetivo do estudo foi determinar se a Gordura intra Abdominal medida pela Ultra-Sonografia (GAUS) em homens e mulheres tem associação com os fatores de risco cardiovascular, comparar essa medida com a circunferência abdominal e diâmetro sagital para precisar qual destes métodos é o melhor preditor de risco cardiovascular (CV), e tentar encontrar um ponto de corte para GAUS que possa definir maior risco de doença $\mathrm{CV}$. Cento e noventa e um homens sadios e 231 mulheres sadias foram submetidas à medidas antropométricas, medidas de pressão arterial sistêmica e dosagens laboratoriais para colesterol, HDL, triglicerídeos e glicemia. A ultra-sonografia intra-abdominal foi realizada para medir espessura visceral. A amostra foi dividida em três grupos de acordo com a presença dos seguintes fatores de risco cardiovascular: 1) grupo de moderado risco - presença de dois ou mais dos fatores: col $>200 \mathrm{mg} / \mathrm{dL}$, HDL-colesterol < 45mg/dL, TG > 200mg/dL, Glic > 126mg/dL, PAs $>140 \mathrm{mmHg}$. PAd $>90 \mathrm{mmHg}$; 2 ) grupo de alto risco com dois ou mais dos fatores: total col $>240 \mathrm{mg} / \mathrm{dL}$, HDL-colesterol $<35 \mathrm{mg} / \mathrm{dL}, \mathrm{TG}>$ $200 \mathrm{mg} / \mathrm{dL}+\mathrm{HDL}<35 \mathrm{mg} / \mathrm{dL}$, Glic $>126 \mathrm{mg} / \mathrm{dL}$, PAs $>140 \mathrm{mmHg}$, PAd $>$ $90 \mathrm{mmHg}$;) grupo sem risco: com um ou nenhum dos fatores de risco presentes. Os resultados mostraram que a GAUS tem associação com todos os fatores de risco cardiovascular e tem melhor especificidade $e$ acurácia que a circunferência abdominal e o diâmetro sagital. $O$ valor de $7 \mathrm{~cm}$ para ambos os sexos fol o ponto de corte para GAUS para predizer risco moderado; 8 e $9 \mathrm{~cm}$ foram os pontos de cortes obtidos para GAUS para predizer alto risco CV em homens e mulheres, respectivamente. A GAUS é um método útil de determinar espessura visceral e parece ser capaz de predizer risco cardiovascular. (Arq Bras Endocrinol Metab 2000;44/1: 49-56)
\end{abstract}

Unitermos: Gordura intra abdominal; Ultra-sonografia; Risco cardiovascular

\begin{abstract}
The objective of our study was to determine if Intra Abdominal Thickness measured by Ultrasonography (IATU) has correlation with cardiovascular risk factors, and if it is a better predictor of cardiovascular risk than waist circumference and sagittal diameter. Finally we tried to find a cut off point for abdominal thickness measured by ultrasonography to predict cardiovascular disease (CVD). One hundred ninety one healthy men and 231 healthy women were submitted to anthropometric evaluation and blood pressure measure. Blood samples were drawn for total plasma cholesterol, HDL, triglyceride and glucose determination. Intra-abdominal ultrasonography was performed to measure abdominal thickness. The population was divided in three groups according to the presence of CVD risk factors: 1) moderately risk group (MR) with two or more of the following: total chol $>200 \mathrm{mg} / \mathrm{dL}, \mathrm{HDL}$-cholesterol $<45 \mathrm{mg} / \mathrm{dL}$, TRIG > $200 \mathrm{mg} / \mathrm{dL}$, Glu > 126 mg/DI, SBP > 140mmHg. DBP > 90mmHg; 2) high risk group (HR) with two or more of the indicated: total chol $>240 \mathrm{mg} / \mathrm{dl}, \mathrm{HDL}$ cholesterol $<35 \mathrm{mg} / \mathrm{dl}$, TRIG > 200mg/dl + HDL-cholesterol $<35 \mathrm{mg} / \mathrm{dl}$, Glu
\end{abstract}

\section{artigo original}

\author{
Claudia C. Leite \\ Daniella Matsuda \\ Bernardo L. Wajchenberg \\ Giovanni G. Cervi \\ Alfredo Halpern
}

Grupo de Obesidade da Disciplina de Endocrinologia e Metabologia do Hospital das Clinicas da Faculdade de Medicina da Universidade São Paulo e Departamento de Radiologia do Instituto do Coracãa da Faculdade de Medicina da Universidade São Paulo. 
$>126 \mathrm{mg} / \mathrm{dl}, \mathrm{SBP}>140 \mathrm{mmHg}, \mathrm{DBP}>90 \mathrm{mmHg}$; 3) no risk group (NR) - with only one or none of the risk factors indicated in the moderate and high group. The results showed that IATU has association with all cardiovascular risk factors and has better specificity and accurately for CVD risk than waist circumference and sagittal diameter. The values of $7 \mathrm{~cm}$ for both sexes was the cut off lengths obtained by IATU to predict moderate $\mathrm{CV}$ risk; 8 and $9 \mathrm{~cm}$ were the cut off length obtained by IATU to predict high CV risk for men and for women respectively. The IATU is a useful way to determine visceral fat and seems to be predictive of cardiovascular risk. (Arq Bras Endocrinol Metab 2000;44/1: 49-56)

Keywords: Intra abdominal thickness; Ultrasonography: Cardiovascular risk

O Acúmulo de tecido adiroso visceral está relacionado com anormalidades metabólicas, as quais contribuem para o aumento do risco de doença cardiovascular (DCV) (1-6). O limite mínimo de gordura visceral ayaliado pela Tomografia Computadorizada (TC), a nível de L4-L.5, acima do qual as alteraçōes metabólicas são nitidamente observadas, ć $110 \mathrm{~cm}^{2}$ para mulheres de acordo com Willians e col. (7); enquanto que para autores como Després and Lamarche (8), o valor de $100 \mathrm{~cm}^{2}$ tanto para homens como para mulheres já está associado a significantes alterações de risco para doença cardiovascular, e que profunda deterioração metabólica é observada com valores $>130 \mathrm{~cm}^{2}$. TC é o método padrão-ouro para avaliação da adiposidade visceral, mas não pode ser usado como um teste de rotina por ser dispendioso e submeter o indivíduo a radioatividade (9-11). A relaçào cintura-quadril, a circunferência abdominal e o diâmetro sagital são alguns dos métodos antropométricos usados para aferir gordura intra-abdominal, mas têm baixa acurácia (12-14). A ultra-sonografia (US) tem sido usada para mensurar espessura visceral desde 1990, tem as vantagens de ser de fácil manuseio, baixo custo, não envolver exposição à irradiação e ter alta correlação com a $\mathrm{TC}(\mathrm{r}=0,67)$ (15). Embora alguns autores tenham descrito resultados não satisfatórios com a ultra-sonografia (16-17), acreditamos que a divergência na metodologia e a falta de preparo dos operadores seja a explicação para o fato. O objetivo deste estudo é correlacionar espessura da gordura intra-abdominal medida pela ultra-sonografia (GAUS) com os fatores de risco CV, comparar a ultrasonografia com as medidas antropométricas (circunferência abdominal c diâmetro sagital) para definir quual o melhor método como preditor de risco CV e determinar um ponto de corte que possa classificar o indivíduo com maior risco $\mathrm{CV}$.

\section{MATERIAL E MÉTODOS}

Foram estudados 231 mulheres e 191 homens voluntários e sadios, selecionados aleatoriamente, com idades entre 20 e 60 anos c Índice de Massa Corpórea (IMC) entre $20 \mathrm{c} 40 \mathrm{~kg} / \mathrm{m}^{2}$. O critério de exclusão cra a presença de cvento cardiovascular prévio (infarto agudo do miocárdio, angina pectoris, AVC). Todos os participantes assinaram o Termo de Consentimento conforme normas da comissão de ética do Hospital das Clínicas da FMUSP. Três grupos étnicos foram incluídos: caucasianos, pardos e negros. O IMC foi subdividido $\mathrm{cm}$ três escalas $\left(20-24,9 \mathrm{~kg} / \mathrm{m}^{2}, 25-29,9 \mathrm{~kg} / \mathrm{m}^{2}\right.$, $\left.30-40 \mathrm{~kg} / \mathrm{m}^{2}\right)$, assim como as idades (20-35 anos, 3650 anos, > 51 anos). As medidas antropométricas foram realizadas no dia do primeiro atendimento pelo mesmo observador: peso, altura, IMC, circunferência abdominal (menor diâmetro entre a última costela $\mathrm{e}$ crista ilíaca, cstando o indivíduo $\mathrm{cm}$ pé) (18) e diâmetro sagital (aferido com o indivíduo deitado e usando uma régua de madeira, que mede do dorso do indivíduo em contato com a superfícic ao ponto mais elevado do abdômen) (19). A pressão arterial (PAs e PAd) foi medida no braço esquerdo, a nível do coração, após 5 minutos do indivíduo em repouso e sentado. Usamos fita milimetrada para corrigir a medida auscultatória com o diâmetro da circunferência do braço (20). A média entre duas medidas de PA foi usada no estudo (2l).

A espessura de gordura intra-abdominal foi medida pela ultra-sonografia considerando a distância entre a parede posterior do músculo reto abdominal e parede posterior da aorta na região da bifurcação da aorta abdominal, na linha xifo-umbilical, usando aparelho Toshiba Sonolayer SSA-250A com transdutor de $3,75 \mathrm{MHz}$. A espessura visceral e a espessura subcutânea foram determinadas diretamente de imagens congeladas na tela (22). Todos os indivíduos estavam em jejum de 6h e os exames foram realizados pelo mesmo observador (DM). O coeficiente de variação intra-indivíduo foi de $6,5 \%$ para espessura visceral e $1 \%$ para espessura subcutânca em 20 indivíduos avaliados com 24h de diferença.

Amostras de sangue para dosagens bioquímicas foram realizadas $\mathrm{cm}$ dia diferente da consulta, mas com intervalo máximo de sete dias, pela manhã c com o indivíduo em jejum de 12 horas. Foram dosados: colesterol total, HDL-colesterol, triglicerídeos e glicemia. Glicose plasmática foi avaliada pelo glucose analyzer; Colesterol total, HDL-colesterol c triglicerídeos foram determinados pelo método enzimático calorimétrico (aparelho Cobas Integra Plus, 
comercial kit do Roche Diagnostic System Inc.). O coeficiente de variação intra e inter-ensaio apresentou variação < 5\% para todas as dosagens.

Os grupos masculino e feminino foram subdivididos em três grupos de acordo com a presença dos fatores de risco $\mathrm{CV}:$ 1) Grupo de moderado risco: presença de dois ou mais dos seguintes fatores: colesterol $>200 \mathrm{mg} / \mathrm{dL}, \mathrm{HDL}<45 \mathrm{mg} / \mathrm{dL}$, triglicerídeos > $200 \mathrm{mg} / \mathrm{dL}$, Glic > $126 \mathrm{mg} / \mathrm{dL}$, PAs > $140 \mathrm{mmHg}$, PAd $>90 \mathrm{mmHg}$; 2) Grupo de alto risco: dois ou mais dos fatores presentes: colesterol $>240 \mathrm{mg} / \mathrm{dL}, \mathrm{HDL}<$ $35 \mathrm{mg} / \mathrm{dL}$, triglicerídeos $>200 \mathrm{mg} / \mathrm{dL}+\mathrm{HDL}<$ $35 \mathrm{md} / \mathrm{dL}$, Glicemia $>126 \mathrm{mg} / \mathrm{dL}$, PAs $>140 \mathrm{mmHg}$, PAd $>90 \mathrm{mmHg}$; 3) Grupo sem risco: com um ou nenhum dos fatores descritos nos dois grupos anteriores. Os fatores de risco $\mathrm{CV}$ obedeceram os critérios recomendados pelo National Cholesterol Education Program (NCEP 1993) (23).

Para comparação de médias usamos o teste t-Student. Curva ROC (Receiver operating characteristic) e regressão logística foram usadas para encontrar o ponto de corte da GAUS. Para as associações entre os fatores de risco para doença $\mathrm{CV}$ e os dados clínicos e antropométricos usamos o Chi-square test e de Fisher (24). Consideramos significantes valores de $p$ menores que 0.05 .

\section{RESULTADOS}

As características clínicas dos 422 participantes estudados estão na Tabela 1 . Oitenta e nove homens $(46,5 \%)$ e 104 mulheres $(59,1 \%)$ foram classificados como sem risco cardiovascular, 68 homens $(35,6 \%)$ e 72 mulheres $(40,9 \%)$ foram considerados com risco moderado, e 34 homens $(17,8 \%)$ e 55 mulheres $(34,6 \%)$ pertenciam ao grupo de alto risco (Tabelas $2 \mathrm{~A}$ e $2 \mathrm{~B}$ ). O estudo estatístico mostrou associação entre o risco de ocorrência de doença CV e os valores médios de GAUS ( $\mathrm{p}=<0,001$, para ambos os grupos de risco). As médias e desvios padrão (dp) da GAUS em mulheres dos grupos sem risco, risco moderado e alto risco foram: $6,3 \mathrm{~cm}(\mathrm{dp} \pm$ $2,4 \mathrm{~cm}), 7,4 \mathrm{~cm}(\mathrm{dp} \pm 1,97 \mathrm{~cm})$ e $8,5 \mathrm{~cm}(\mathrm{dp} \pm 2,6 \mathrm{~cm})$, respectivamente. A GAUS média para os homens sem risco da ocorrência de doença CV foi igual a $7,0 \mathrm{~cm}(\mathrm{dp}$ $\pm 2,3 \mathrm{~cm})$, risco moderado $8,1 \mathrm{~cm}(\mathrm{dp} \pm 2 \mathrm{~cm})$ e alto risco $9,3 \mathrm{~cm}(\mathrm{dp} \pm 2,6 \mathrm{~cm})$.

A associação entre os fatores de risco moderado isoladamente (PAs, PAd, COL, HDL, TRIG) e a GAUS mostrou-se significativa em mulheres para a PAs $(\mathrm{p}=0,003)$ e triglicerídeos $(\mathrm{p}=0,047)$. Nenhuma muther neste grupo apresentou PAd $>90 \mathrm{mmHg}$. Todos os fatores de risco alto no grupo feminino apresentaram associação estatística com a GAUS, PAs $(\mathrm{p}=<0,001)$, PAd $(\mathrm{p}=0,003)$, colesterol $(\mathrm{p}=0,049)$, HDL $(\mathrm{p}=$ $0,010)$, HDL e triglicerídeo $(\mathrm{p}=0,010)$ e com glicemia $(\mathrm{p}=<0,001)$. No grupo masculino houve associação estatística significante entre os fatores de risco moderado e a GAUS apenas para a PAs $(\mathrm{p}=<0,001)$. Entre os fatores de risco alto observou-se associação estatística com a GAUS: $o$ colesterol $(\mathrm{p}=0,001)$, Glicemia $(\mathrm{p}=$ $<0,001)$, HDL + TRIG $(p=0,009)$, e PAs $(p=<0,001)$ (Tabela 3 ). Os valores de GAUS são sempre maiores quando o fator de risco está presente.

Tabela 1. Demonstração dos dados clínicos da amostra de 191 homens e 231 mulheres estudados.

\begin{tabular}{lccc}
\hline $\begin{array}{l}\text { Variavél } \\
\text { Clínica }\end{array}$ & Classificação & $\begin{array}{c}\text { Homens } \\
(\mathrm{n} / \%)\end{array}$ & $\begin{array}{c}\text { Mulheres } \\
(\mathrm{n} / \%)\end{array}$ \\
Idade & $20-35$ & $88 / 46$ & $79 / 34,2$ \\
(anos) & $36-50$ & $90 / 47,2$ & $109 / 47,2$ \\
& $>51$ & $13 / 7$ & $43 / 18,6$ \\
Raça & Branca & $91 / 47,6$ & $121 / 52,4$ \\
& Negra & $19 / 9,9$ & $28 / 12,7$ \\
IMC & Parda & $81 / 42,4$ & $82 / 35,5$ \\
$\left(\mathrm{~kg} / \mathrm{m}^{2}\right)$ & $20-24,9$ & $38 / 19,9$ & $26 / 11,3$ \\
& $25-29,9$ & $80 / 41,9$ & $47 / 20,3$ \\
Tabagistas & $30-40$ & $73 / 38,2$ & $158 / 68,4$ \\
Menopausadas & & $50 / 26,1$ & $40 / 17,3$ \\
\hline
\end{tabular}

$\mathrm{n}=$ número, $\%=$ porcentagem 
Tabela 2A. Perfil clínico dos três grupos de risco (sexo masculino).

\begin{tabular}{|c|c|c|c|}
\hline \multirow[t]{2}{*}{ Caracter } & $\begin{array}{c}\text { Grupo Sem } \\
\text { Risco } \\
(n=89)\end{array}$ & $\begin{array}{c}\text { Grupo de } \\
\text { Risco } \\
\text { Moderado } \\
(n=68)\end{array}$ & $\begin{array}{c}\text { Grupo de Alto } \\
\text { Risco } \\
(n=34)\end{array}$ \\
\hline & \multicolumn{3}{|c|}{$\begin{array}{c}n^{\circ} \text { de individuos da } \\
\text { amostragem }\end{array}$} \\
\hline $\begin{array}{l}\text { Idade (anos): } \\
\quad 20-35 \\
36-50 \\
>51 \text { anos }\end{array}$ & $\begin{array}{l}51 \\
36 \\
02\end{array}$ & $\begin{array}{l}30 \\
36 \\
02\end{array}$ & $\begin{array}{l}07 \\
28 \\
09\end{array}$ \\
\hline $\begin{array}{l}\text { Raça: } \\
\text { Brancos } \\
\text { Pardos } \\
\text { Negros }\end{array}$ & $\begin{array}{l}42 \\
40 \\
07\end{array}$ & $\begin{array}{l}33 \\
28 \\
07\end{array}$ & $\begin{array}{l}16 \\
13 \\
05\end{array}$ \\
\hline $\begin{array}{c}\text { IMC }\left(\mathrm{kg} / \mathrm{m}^{2}\right) \\
20-24,9 \\
25-29,9 \\
30-40\end{array}$ & $\begin{array}{l}25 \\
24 \\
40\end{array}$ & $\begin{array}{l}08 \\
30 \\
30\end{array}$ & $\begin{array}{l}05 \\
19 \\
10\end{array}$ \\
\hline Tabagismo & 20 & 23 & 07 \\
\hline
\end{tabular}

Tabela 2B. Perfil clínico nos três grupos de risco (sexo feminino)

\begin{tabular}{|c|c|c|c|}
\hline Caracter & $\begin{array}{c}\text { Grupo sem } \\
\text { Risco } \\
(n=104)\end{array}$ & $\begin{array}{c}\text { Grupo de Risco } \\
\text { Moderado } \\
(n=72)\end{array}$ & $\begin{array}{c}\text { Grupo de Alto } \\
\text { Risco } \\
(n=55)\end{array}$ \\
\hline & & $\begin{array}{l}\mathrm{n}^{\circ} \text { de indivíduos do } \\
\text { amostragem }\end{array}$ & \\
\hline $\begin{array}{c}\text { Idade (anos) } \\
20-35 \\
36-50 \\
>57^{\circ}\end{array}$ & $\begin{array}{l}51 \\
42 \\
11\end{array}$ & $\begin{array}{l}22 \\
40 \\
10\end{array}$ & $\begin{array}{l}06 \\
27 \\
22\end{array}$ \\
\hline $\begin{array}{l}\text { Raça: } \\
\text { Branca } \\
\text { Parda } \\
\text { Negra }\end{array}$ & $\begin{array}{l}52 \\
40 \\
12\end{array}$ & $\begin{array}{l}44 \\
23 \\
05\end{array}$ & $\begin{array}{l}25 \\
19 \\
11\end{array}$ \\
\hline $\begin{array}{c}\mathrm{IMC}\left(\mathrm{Kg} / \mathrm{m}^{2}\right) \\
20-24,9 \\
25-29,9 \\
30-40\end{array}$ & $\begin{array}{l}17 \\
25 \\
62\end{array}$ & $\begin{array}{l}04 \\
19 \\
49\end{array}$ & $\begin{array}{l}05 \\
03 \\
47\end{array}$ \\
\hline $\begin{array}{l}\text { Tabagismo } \\
\text { Menopausa }\end{array}$ & $\begin{array}{l}17 \\
12\end{array}$ & $\begin{array}{l}16 \\
10\end{array}$ & $\begin{array}{l}07 \\
17\end{array}$ \\
\hline
\end{tabular}

Tabela 3. Associação dos fatores de risco cardiovascular isoladamente e a gordura intra abdominal medida pela ultra-sonografia (GAUS) em cada grupo de risco (Médias e desvios-padrão da GAUS).

\begin{tabular}{|c|c|c|c|c|c|}
\hline $\begin{array}{l}\text { Fatores de risco isolados } \\
\text { PAs }>140 \mathrm{mmHg}\end{array}$ & $\begin{array}{l}\text { Homens } \\
\text { Ausente } \\
\text { Presente }\end{array}$ & $\begin{array}{c}\text { Risco Moderado } \\
P^{*} \\
6,87 \pm 2,00 \\
8,04 \pm 2,26\end{array}$ & $\begin{array}{l}\text { Mulheres } \\
<0,001\end{array}$ & $\begin{array}{c}P^{*} \\
6,43 \pm 2,09 \\
8,02 \pm 2,70\end{array}$ & 0,003 \\
\hline PAd $>90 \mathrm{mmHg}$ & $\begin{array}{l}\text { Ausente } \\
\text { Presente }\end{array}$ & $\begin{array}{l}7,32 \pm 2,15 \\
8,19 \pm 2,41 .\end{array}$ & 0,082 & - & - \\
\hline Colesterol $>200 \mathrm{mg} / \mathrm{dL}$ & $\begin{array}{l}\text { Ausente } \\
\text { Presente }\end{array}$ & $\begin{array}{l}7,48 \pm 2,33 \\
7,44 \pm 2,73\end{array}$ & 0,920 & $\begin{array}{l}0,64 \pm 2,60 \\
6,85 \pm 1,94\end{array}$ & 0,552 \\
\hline Triglicerideo $>200 \mathrm{mg} / \mathrm{DI}$ & $\begin{array}{l}\text { Ausente } \\
\text { Presente }\end{array}$ & $\begin{array}{l}7,41 \pm 2,33 \\
7,66 \pm 1,37\end{array}$ & 0,480 & $\begin{array}{l}6,63 \pm 2,30 \\
7,83 \pm 1,98\end{array}$ & 0,047 \\
\hline $\mathrm{HDL}<45 \mathrm{mg} / \mathrm{DI}$ & $\begin{array}{l}\text { Ausente } \\
\text { Presente }\end{array}$ & $\begin{array}{c}7,49 \pm 2,19 \\
4,20(1 \text { pct. })\end{array}$ & 0,958 & $\begin{array}{l}6,56 \pm 2,33 \\
6,92 \pm 2,26\end{array}$ & 0,294 \\
\hline Fatores de risco isolados & Homens & $\begin{array}{c}\text { Risco Alto } \\
P\end{array}$ & Mulheres & $p$ & \\
\hline PAs $>140 \mathrm{mmHg}$ & $\begin{array}{l}\text { Ausente } \\
\text { Presente }\end{array}$ & $\begin{array}{l}7,29 \pm 2,40 \\
8,86 \pm 2,80\end{array}$ & 0,004 & $\begin{array}{l}6,31 \pm 2,29 \\
8,52 \pm 2,80\end{array}$ & $<0,001$ \\
\hline PAd $>90 \mathrm{mmHg}$ & $\begin{array}{l}\text { Ausente } \\
\text { Presente }\end{array}$ & $\begin{array}{l}7,60 \pm 2,53 \\
8,42 \pm 2,81\end{array}$ & 0,202 & $\begin{array}{l}6,75 \pm 2,58 \\
8,37 \pm 2,72\end{array}$ & 0,003 \\
\hline Colesterol $>240 \mathrm{mg} / \mathrm{dL}$ & $\begin{array}{l}\text { Ausente } \\
\text { Presente }\end{array}$ & $\begin{array}{l}7,34 \pm 2,47 \\
9,29 \pm 2,54\end{array}$ & 0,001 & $\begin{array}{l}6.79 \pm 2.62 \\
7.74 \pm 2.72\end{array}$ & 0,049 \\
\hline $\mathrm{HDL}<35 \mathrm{mg} / \mathrm{dL}$ & $\begin{array}{l}\text { Ausente } \\
\text { Presente }\end{array}$ & $\begin{array}{l}7,61 \pm 2,57 \\
7,91 \pm 2,67\end{array}$ & 0,607 & $\begin{array}{l}6,78 \pm 2,54 \\
8,21 \pm 2,97\end{array}$ & 0,010 \\
\hline $\begin{array}{l}\text { Triglicerídeo > } 200 \mathrm{mg} / \mathrm{dL} \\
\text { e } \mathrm{HDL}<35 \mathrm{mg} / \mathrm{dL}\end{array}$ & $\begin{array}{l}\text { Ausente } \\
\text { Presente }\end{array}$ & $\begin{array}{l}7,52 \pm 2,53 \\
9,96 \pm 2,30\end{array}$ & 0,009 & $\begin{array}{l}6,86 \pm 2,61 \\
8,77 \pm 2,75\end{array}$ & 0,010 \\
\hline Glicemia > 126mg/dL & $\begin{array}{l}\text { Ausente } \\
\text { Presente }\end{array}$ & $\begin{array}{c}7,42 \pm 2,40 \\
10,55 \pm 2,93\end{array}$ & $<0,001$ & $\begin{array}{l}6,85 \pm 2,59 \\
9,78 \pm 2,35\end{array}$ & $<0,001$ \\
\hline
\end{tabular}


O estudo por regressão logística do grupo feminino mostrou uma correlação da GAUS como preditor de risco $\mathrm{CV}$ moderado $(\mathrm{p}=0,0017 \mathrm{cOR}=$ $1,25 \mathrm{com}$ intervalo de confiança (IC) a $95 \%$ de 1,09 1,45 ). Isto significa que, a cada um centímetro a mais de espessura intra-abdominal no grupo feminino, o risco moderado de doença CV aumenta de 1,25 vezes. O grupo de risco alto apresentou a mesma correlação estatística $(p=0,001) \operatorname{com} O R=1,40(\mathrm{IC}$ a 95\%: 1,2l-1,63). Também no grupo masculino a GAUS foi significantemente maior nos homens dos grupos de risco em relação aos sem risco para desenvolver doença CV. A GAUS mostrou ser um fator de risco nos homens para predizer risco moderado de doença $C V(p=0,005)$ e OR $=1,25$ (IC a 95\%: 1,07 a 1,47$)$ e para o alto risco $(\mathrm{p}=0,0001)$, com $\mathrm{OR}=$ 1,47 (IC a $95 \%: 1,23$ a 1,77 ).

$\mathrm{Na}$ busca de um valor de corte que pudesse separar as mulheres com maior ou menor risco levando em conta a GAUS, também usamos a regressão logística e encontramos o valor de $7 \mathrm{~cm}$ como limite superior da normalidade e melhor preditor de risco $\mathrm{CV}$ moderado $(\mathrm{p}=0,0005)$ e OR $=3,01$ (IC a $95 \%$ de 1,61-5,63). Com este corte temos uma sensibilidade de $58,3 \%$ e especificidade de $68,3 \%$. No grupo classificado como de alto risco, o corte encontrado foi de $8 \mathrm{~cm}$ de GAUS $(\mathrm{p}=0,0008)$, c OR $=3,27$ (IC a $95 \%$ : $1,64-6,60)$. Este valor de corte confere uma sensibilidade de $50,9 \%$ e uma especificidade de $76 \%$. No grupo masculino, o valor de corte encontrado também foi de
$7 \mathrm{~cm}(\mathrm{p}=0,0027)$ para o risco moderado e $\mathrm{OR}=2,86$ (IC a $95 \%: 1,44$ a 5,68$)$ e $9 \mathrm{~cm}(\mathrm{p}=0,0001) \mathrm{com}$ OR de 5,55 (IC a 95\%:2,3-13,3) para o alto risco. A este nível de corte a sensibilidade e especificidade encontradas foram de $72 \%$ e $53 \%$ e de $53 \%$ e $83 \%$, respectivamente, para os grupos de moderado e alto risco. Os valores de GAUS de corte encontrado têm associaçào cstatística com todos fatores de risco $\mathrm{CV}$.

Nos homens, levando $\mathrm{cm}$ conta os fatores prognósticos de doença CV como idade, IMC, circunferência abdominal, diâmetro sagital e GAUS, e estudando-os individualmente e independentemente, por um processo de seleção das variáveis, a GAUS mostrou-se importante marcador de risco mesmo sem a influchncia dos outros fatores $(p=0,03)$. Para cstc modelo de estudo usamos o valor de corte de GAUS de $7 \mathrm{~cm}$. No grupo feminino, a GAUS também manteve-se como importante sinalizadora para o risco cardiovascular $(\mathrm{p}=0,01)$ (Tabela 4$)$.

Quando estudamos a GAUS, CA e DS, encontramos uma correlação positiva entre estas medidas. Quanto maior o risco para DCV, maiores as médias de CA, DS e GAUS, mas apenas a GAUS apresentou médias diferentes para os trếs grupos (Tabela 5)

Com a finalidade de comparar a GAUS com a CA e DS como preditores de risco $\mathrm{CV}$, analisamos a sensibilidade, especificidade e acurácia de cada medida. O resultado obtido mostrou que a GAUS apresenta melhores valores de especificidade e acurácia no grupo de alto risco (Tabela 6).

Tabela 4. Capacidade de predição de risco cardiovascular entre os fatores prognósticos de DCV.

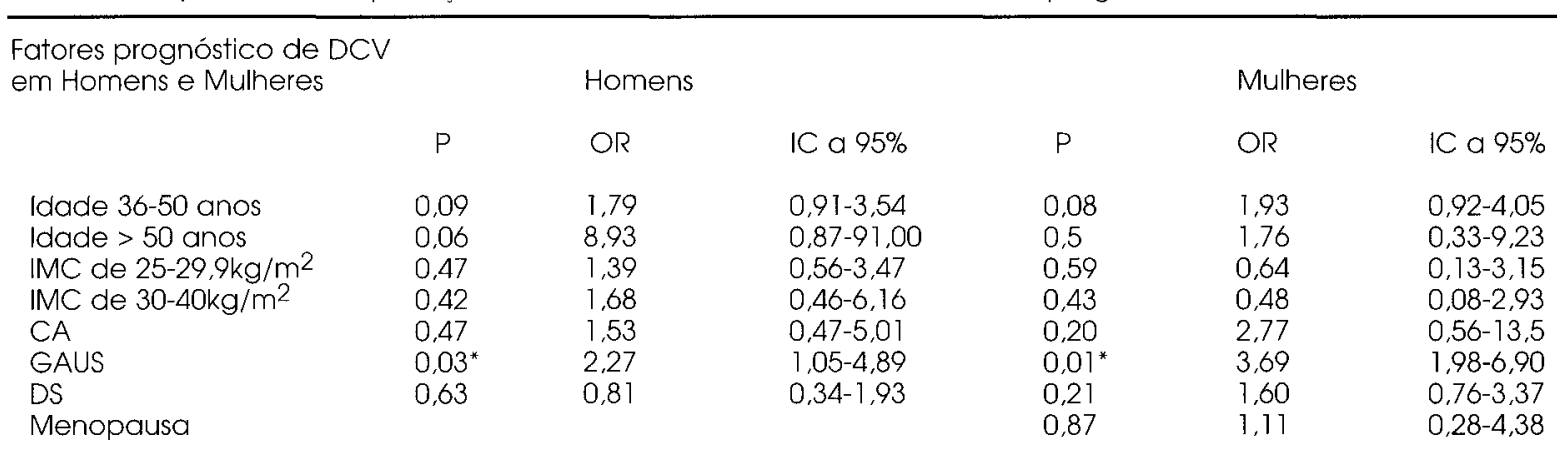

$C A=$ circunferência abdominal

DS = diâmetro sagital

GAUS = gordura intra abdominal medida por ultra-sonografia

$\mathrm{OR}=$ odds ratio (razão de chance de um determinado evento)

$\mathrm{IC}=$ intervalo de confiança

DVC $=$ doença cardiovascular

Considerado significante $\mathrm{P}=<0,05$ 
Espessura Intra-Abdominal por US e Fatores de Risco

Leite et al.

Tabela 5. Valores das médias da GAUS, circunferência abdominal e diâmetro sagital em mulheres, nos três grupos de risco.

\begin{tabular}{|c|c|c|c|c|}
\hline Grupo de Risco & $\begin{array}{l}\text { Números } \\
\text { Mulheres }\end{array}$ & $\begin{array}{l}\text { Método } \\
\text { Medida }\end{array}$ & Médias & DESVIO PADRÃO \\
\hline Sem Risco & 103 & $\begin{array}{c}\text { CA } \\
\text { DS } \\
\text { GAUS }\end{array}$ & $\begin{array}{r}94,79 \\
22,47 \\
6,25\end{array}$ & $\begin{array}{r}12,65 \\
4,14 \\
2,40\end{array}$ \\
\hline Moderado & 72 & $\begin{array}{c}\text { CA } \\
\text { DS } \\
\text { GAUS }\end{array}$ & $\begin{array}{r}102,33 \\
23,60 \\
7,41\end{array}$ & $\begin{array}{r}12,21 \\
3.65 \\
1,97\end{array}$ \\
\hline Alto & 56 & $\begin{array}{c}\text { CA } \\
\text { DS } \\
\text { GAUS }\end{array}$ & $\begin{array}{r}104.65 \\
25.36 \\
8.47\end{array}$ & $\begin{array}{r}10,83 \\
4,46 \\
2,56\end{array}$ \\
\hline
\end{tabular}

$C A=$ circunferência abdominal

DS = diâmetro sagital

GAUS = ESPESSURA intra-abdominal medida pela ultra-sonografia

Comparação entre as médias de GAUS, CA e DS nos três grupos de risco em mulheres.

\begin{tabular}{lrccc}
\hline Método de Medida & Valores de $\mathrm{p}$ & Alto $x$ moderado & Alto $x$ sem risco & Moderado $\times$ sem risco \\
Ca & 0,0001 & $N S$ & $P<0,05$ & $P<0,05$ \\
Gaus & 0,0001 & $P<0,05$ & $P<0,05$ & $P<0,05$ \\
Ds & 0,0004 & $N S$ & $P<0,05$ & $N S$ \\
\hline
\end{tabular}

NS = sem significância estatística

$P=$ nivel descritivo de probabilidade do teste t-Student $(p=<0,05)$

Tabela 6. Valores de sensibilidade, especificidade e acurácia da GAUS, circunferência abdominal e diâmetro sagital nos dois grupos de risco.

\begin{tabular}{|c|c|c|c|c|c|c|}
\hline \multirow[b]{2}{*}{$\begin{array}{l}\text { Homens } \\
\text { Gaus } \\
\text { Ca } \\
\text { Sagital }\end{array}$} & \multicolumn{3}{|c|}{ Risco Moderado } & \multicolumn{3}{|c|}{ Risco Alto } \\
\hline & $\begin{array}{c}\text { Sensib. } \\
59,4 \\
71,9 \\
76,3\end{array}$ & $\begin{array}{c}\text { Esprec. } \\
59,6 \\
52,8 \\
47,1\end{array}$ & $\begin{array}{c}\text { Acurácia } \\
59,5 \\
60,8 \\
58,9\end{array}$ & $\begin{array}{c}\text { Sensib. } \\
52,9 \\
52,6 \\
43,8\end{array}$ & $\begin{array}{c}\text { Esprec. } \\
82,0 \\
77,5 \\
80,5\end{array}$ & $\begin{array}{c}\text { Acurácia } \\
74,0 \\
70,1 \\
70,6\end{array}$ \\
\hline \multicolumn{7}{|l|}{ Mulheres } \\
\hline $\begin{array}{l}\text { Gaus } \\
\text { Ca } \\
\text { Sagital }\end{array}$ & $\begin{array}{l}54,2 \\
97,2 \\
80,6\end{array}$ & $\begin{array}{l}68,3 \\
14,1 \\
34,4\end{array}$ & $\begin{array}{l}62,5 \\
48,3 \\
52,5\end{array}$ & $\begin{array}{l}50,9 \\
89,1 \\
66,7\end{array}$ & $\begin{array}{l}76.0 \\
30,8 \\
59,4\end{array}$ & $\begin{array}{l}67.3 \\
50,9 \\
61,8\end{array}$ \\
\hline
\end{tabular}

\section{DISCUSSÃO}

A medida da espessura da gordura visceral é considerada um importante indicador do risco cardiovascular, devido às alterações metabólicas decorrentes deste depósito gorduroso. Usamos rotineiramente para definir obesidade central, medidas antropométricas (como a circunferência abdominal e o diâmetro sagital) por serem de fácil aplicação prática. Estes métodos têm baixa acurácia, principalmente por não separar gordura intra-abdominal da subcutânea.

A Tomografia Computadorizada é considerada método padrão-ouro para quantificar volume visceral, mas não pode ser usada rotineiramente pelos inconvenientes impostos pelo exame (25). A ultra-sonografia por ter baixo custo, fácil acesso e ser isenta de efeitos adversos ao paciente, parece ser um exame útil e de boa aplicabilidade para mensurar espessura visceral (26). Armellini et al. (27) desenvolvem, desde 1990, trabalhos comprovando a eficácia da ultra-sonografia e sua boa correlação com a TC em mensurar espessura intra-abdominal $(r=0,669, p<0,001)$. No nosso estudo, a GAUS mostrou ter boa associação com os fatores de risco $\mathrm{CV}$, ficando mais evidente essa associação nos grupos classificados como de alto risco. Também constatamos ter a GAUS melhor especificidade e 
acurácia que a circunferência abdominal e o diâmetro sagital, provavelmente por ser a ultra-sonografia mais precisa que estas medidas antropométricas em quantificar espessura visceral. Conseguimos encontrar um valor de corte para ambos os sexos no grupo de moderado risco CV de $7 \mathrm{~cm}$, e para predizer alto risco CV os valores de corte encontrados foram de $8 \mathrm{~cm}$ para as mulheres e $9 \mathrm{~cm}$ para os homens.

\section{CONCLUSŌES}

Concluímos, que a ultra-sonografia parece ser um método útil, prático e melhor que a circunferência abdominal e o diâmetro sagital, para avaliar espessura visceral e risco cardiovascular e valores acima de $7 \mathrm{~cm}$ parecem representar risco cardiovascular para ambos os sexos. Por outro lado, apesar de ser mais específica e com maior acurácia que a CA e DS no grupo de alto risco, a sua avaliação não se mostrou muito superior às mesmas.

\section{REFERÊNCIAS}

1. Kissebah AH, Vydelingum N, Murray RW, Evans DJ, Hartz AJ, Kalkhoff RK, et al. Relation of body fat distribution to metabolic complications of obesity, J Clin Endocrinol Metab 1982;54:254-60.

2. Lapidus L, Bengtsson C, Larsson B, Pennet K, Rybo E, Sjostrom L. Distribution of adipose tissue and risk of cardiovascular disease and death: a 12 year follow up of participants in the population study of women in Gothenburg, Sweden. Br Med J 1984;289:1261-3.

3. Hubert HB, Feinlieb M, McNamara PM, Castelli WP. Obesity as an independent risk factor for cardiovascular disease: a 26-year follow-up of participants in the Framingham heart study. Circulation 1983:67:5-14.

4. Larsson B, Svardsudd K, Welin L, Wilhelmsen L, Bjorntorp $P$. Tibblin $G$. Abdominal adipose tissue distribution, obesity and risk of cardiovascular disease and death: 13year follow-up of participants in the study of men born in 1913. Br Med J 1984:288:1401-4.

5. Micciolo R, Bosello O, Ferrari P, Armellini F. The association of body fat location with haemodynamic and metabolic status in men and women aged $21-60$ years. J Clin Epidemiol 1991:44:591-608.

6. Fujioka S, Matsuzawa Y. Tokunaga K, Tarui S. Contribution of intra-abdominal fat accumulation to the impairment of glucose and lipid metabolism in human obesity. Metabolism 1987:36:54-9.

7. Willians MJ, Hunter GR, Keres-Szabo T, Trueth MS, Snyder $S$. Berland $L$, et al. Intra abdominal adipose tissue cut points related to elevated cardiovascular risk in women. Int J Obes Relat Metab Disord 1996;20:613-7.

8. Després JP, Lamarche B. Effects of diet and physical activity on adiposity and body fat distribution: implications for the prevention of cardiovascular disease. Nutr Res Rev 1993:6:137-59.

9. Borkan GA, Gerzof SG, Robbins AH, Hults DE, Silbert CK,
Silbert JE. Assessment of abdominal fat content by computed tomography. Am J Clin Nutr 1982;36:172-7.

10. Tokunaga K, Matsuzawa $Y$, Ishikawa K, Tarui S. A novel technique for the determination of body fat by computed tomography. Int J Obes 1983:7:437-45.

11. Grauer WO, Moss AA, Cann CE, Golberg HI. Quantification of body fat distribution in the abdomen using computed tomography. Am J Clin Nutr 1984;39:631-7.

12. Després JP, Prud'Homme D, Pouliot MC, Tremblay A, Bouchard C. Estimation of deep abdominal adipose-tissue accumulation from simple anthropometric measurements in men. Am J Clin Nutr 1991:54:471-7.

13. Ross R, Rissanen J, Hudson R. Sensitivity associated with the identification of visceral adipose tissue levels using waist circumference in men and women: effects of weight loss. Int J Obes Relat Metab Disord 1996;20:533-8.

14. Seidell JC, Andres R, Sorkin JD, Muller DC. The sagital waist diameter and mortality in men: the Baltimore Longitudinal Study on aging. Int J Obes Relat Metab Disord 1994:18:61-7.

15. Armellini F, Zamboni M, Rigo L, Todesco T, BergamoAndreis IA, Procacci $C$, et al. The Contribution of sonography to the measurement of intra-abdominal fat. J Clin Ultrasound 1990; 18:563-7.

16. Bellisari A, Roche AF, Siervogel RM. Reliability of b-mode ultrasonic measurements of subcutaneous adipose tissue and intra-abdominal depth: comparisons with skinfold thicknesses. Int J Obes 1993;17:475-80.

17. Van Der Kooy K, Leenen R, Seidell JC, Dewenberg $P$, Visser M. Abdominal diameters as indicators of visceral fat: comparison between magnetic resonance imaging and anthropometry. Br J Nutr 1993:70:47-58.

18. Lohman TG, Roche AF, Martorell R. Anthropometric Standardization Reference Manual. Champaign, IL, Human kinetics 1998 90p

19. Sjostrom $L$ et al. The saggital diameter is a valid marker of the visceral adipose tissue volumen. Progress in Obes Res. 7ed. A. Angel y col. J. Libbey. London 1996.

20. Maxwell $\mathrm{MH}$ et al. Error in blood pressure measurement due to incorrect cuff size in obese patients, Lancet 1982;33-6.

21. Perloff D, Grim C, Flack J. Human blood pressure determination by sphygnomanometry. Circulation 1993:88:2460-7.

22. Tornaghi $G$, Raiteri $R$, Pozzato $C$, Rispoili $A$, Bramani $M$. Cipolat $M$, ef al. Anthropometric or ultrasonic measurements in assessment of visceral fat? A comparative study. Int J Obes Relat Metab Disord 1994; 18:771-5.

23. Summary of the Second Report of the National Cholesterol Education Program (NCEP) Expert Panel on Detection, Evaluation, and Treatment of High Blood Cholesterol in Adults (Adult Treatment Panel II). J Am Med Assoc 1993:269:3015-23.

24. Snedecor GW, Cochran WG. Statistical Methods. $8 \infty e$ dition, lowa State University Press/Ames, 1989 503p

25. Sjostrom L. A computed tomography based multicompartment body composition technique and anthropometric predictions of lean body mass, total and subcutaneous adipose tissue. Int J Obes 1991:15:19-30. 
26. Armellini F, Zamboni $M$, Rigo L, Bergamo-Andreis $I A$, Robbi $R$, Demarchi $M$, et al. Sonography detection of small intra-abdominal fat variations. Int J. Obes $1991 ; 15: 847-52$.

27. Armellini F, Zamboni M, Robbi R, Todesco T, Rigo L, Bergamo-Andreis Al, et al. Total and intra abdominal fat measurements by ultrasound and computerized tomography. Int J Obes 1983:17:209-14.

\section{Endereço para correspondência:}

Claudia Cozer Leite

Dpto. Endocrinologia e Metabologia do Hospital das Clínicas da FMUSP

Av. Dr. Enéas de Carvalho Aguiar $s / n, 8^{\circ}$ andar Prédio dos Ambulatórios - Secretaria da Endocrinologia Fax: (11) 814-9491 